\title{
Experimental design for an enhanced parametric modeling of supercapacitor equivalent circuit model
}

\author{
Ali Mohsen Alsabari, M.K Hassan, Azura CS, Ribhan Zafira \\ Department of Electrical and Electronic Engineering, Universiti Putra Malaysia, Serdang, Selangor, Malaysia
}

\begin{tabular}{l} 
Article Info \\
\hline Article history: \\
Received Mar 22, 2021 \\
Revised Jun 9, 2021 \\
Accepted Jun 15, 2021 \\
\hline
\end{tabular}

\section{Keywords:}

Charge/discharge behaviour

Internal resistance

Neware BTS4000

Parametric modeling

Supercapacitor

Terminal voltage

\begin{abstract}
The modelling of the supercapacitor (SC) plays an important role for the industrial application with many model representations such as electrical, chemical, and electrochemical models. Among one of those models are the equivalent circuit model which has been used to describe the real-time (charging/discharging) operation characteristics of the SC. Apart of its mathematical complexity, the time-consuming experimentally is also a real challenge for obtaining the internal parameters values for the SC. Choices of test equipment with a structure design of experiment also play important criteria affect the accuracy of the model. This research emphasis on a structured of experimental design for SC modelling by using neware battery tester. The experimental exercise to attain internal parameters of the SC are described and discussed in the paper. The findings were benchmarked with an empirical model of previous researchers. The terminal voltage of SC was validated via experiment with maximum relative error of $0.045 \%$. The model successfully reproduce the SC dynamic behavior during the charge/discharge phase which indicates the proposed method and model accuracy.
\end{abstract}

This is an open access article under the CC BY-SA license.

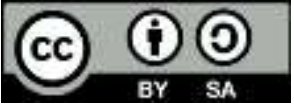

Corresponding Author:

M.K. Hassan

Department of Electrical and Electronic Engineering

Universiti Putra Malaysia

Serdang, Selangor, 43300 Malaysia

Email: khair@upm.edu.my

\section{INTRODUCTION}

Many research cases are carrying out to discover a new sustainable power source answers for conventional power sources in various industrial applications. The energy storage systems (ESS) is considered one of those arrangements as sustainable power sources [1]-[3]. Supercapacitor is one of the main components in ESS which have the attributes of highpower density, long life span whichcan be charged and released in a couple of seconds [4]-[6]. One of the advanced power components in such a capacitor is the electric double-layer capacitors (EDLC) which stores the eneregy by thousands times compared with typical regular capacitor [7]. It comprises of two equal plates that have positive and negative charges isolated by protector as appeared in Figure 1 [8].

Fundamentally, SCs store electrical energy through the development of the double layer capacitor structure at the interface between the anodes and the electrolyte [9]. The uses of supercapacitor can be varied between electric vehicles (EVs), solar/wind power applications remote sensor nodes and hybrid electrical vehicles [10]. Supercapacitors have an essentially lower energy density and higher power density when compared with traditional batteries [11]. Combination of thebattery and supercapacitor has integral characteristics and gives an astounding arrangement that can cover a wide scope of intensity and vitality necessities [12]. 


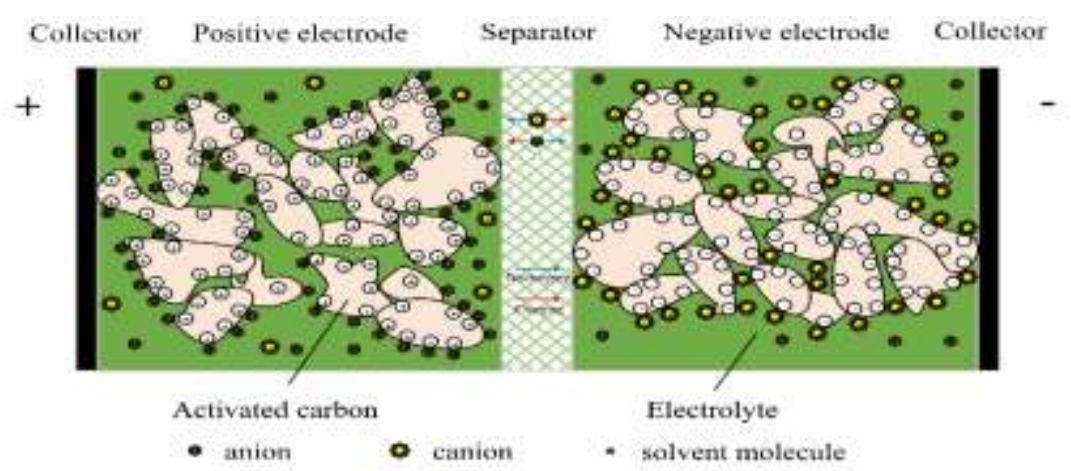

Figure 1. Supercapacitor cell structure

Accordingly, upgrade of intensity quality utilizing batteries and supercapacitors is effectively sought after in the field of sustainable power source and it was shown that this hybridization has lower battery costs, an overall increment in battery life and higher efficiency which may not be fulfilled by the single storage device [13], [14] hence the hybridization was grown effectively in numerous applications like battery electric vehicle, hybrid electric vehicle and uninterruptible power supply as presented in [15]-[17]. In ESSs applications, they ordinarily use SC terminal voltage as the criticism of regulator to accomplish constant control for the SC [18]. Nonetheless, the SC terminal voltage would be influenced by numerous elements, To viably assess the SCs terminal voltage and accomplish the exact control for the SCs in the ESSs, it is pivotal to build up a precise model for the SC [3]. Displaying of SCs has happened to greatest significance when planning and dimensioning SC establishments since it is the best approach to know ahead of time about the conduct and execution of the vitality energy storage devices when applied to specific hybridization [19].

Control systems of supercapacitor or operational boundaries and cutoff points can be acquired from a model, amplifying the lifetime of the capacity and hence accomplishing a more significant level of unwavering quality and competitiveness [20]. Numerous SC models have been distributed in the literature for various purposes, including describing electrical unique behavior, which is of most extreme significance for the industrial applications [21]. The models that describe electrical behavior of SCs can be characterized in three primary classes: electrochemical models, intelligent models and equivalent circuit models. The proposed models vary based on the specific application that has been addressed and intended for convenience in that application [22]. The model should likewise avoid complexity so that it tends to be effortlessly joined into real time controllers [23]. Equivalent circuit models impersonate the electrical behavior of SCs with parametrized capacitors, inductances, and resistors (RLC). They focus on effortlessness, subbing PDEs with customary differential equations (ODEs), which tremendously encourage their usage and make them especially appropriate for industry application investigation and studies [24].

Three subcategories can be considered for equivalent circuit models: RC models, transmission line models, and dynamic models [16]. Model power under transient and differing conditions is of most extreme significance [25]. Nevertheless, the different models of supercapacitor studied in the literature, choosing the model depending on application of the model. In this paper the two branches model were used for electrical behavior applications since it is considered as one of the most widespread model [15]. Even though the chosen model was studied previously, there are many hardware tools used for paramters identification and the method of identifying the internal paratmters of supercapacitor is mathematically complex using least square method. However, in this research case an efficient tool of testing device (neware) only will be used with proposed simple and fast empirical equations for parameter identifications for modeling purpose.

This work emphasis on the terminal voltage dynamics, the calculation of the internal parameters of SC. Not only that, but also the experiments (pulse relaxation) for validation purposes were designed structurally. The robustness tests of the model has been benchmarked with Matlab model and Error analysis validation.The following sections of this paper are organized as; materials and methods, model structure, identification of model parameters, simulation model, results with discussion and conclusion.

\section{MATERIALS AND METHODOLOGY}

\subsection{Model structure}

The model was built in this paper based on two branches circuit which has the following structure precisely displayed in Figure 2. As in Figure 2 the main branch corresponds to the immediate response of the 
supercapacitor during the charge or discharge event in the time range of minutes. In the main branch, R1 is the series resistance and represents the waste power for internal heating on charging and discharging $(\Omega)$.

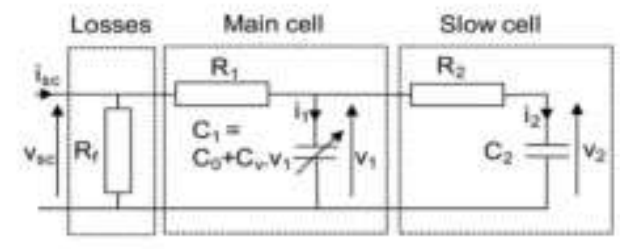

Figure 1. Supercapacitor two branches model [13]

The voltage of the supercapacitor module and other basic relationships described in [6] and [7] can be represented by (1)-(8) as follows:

$$
V_{s c}=N_{s}\left(V_{1}+R_{1} \frac{I s c}{N_{P}}\right)
$$

Where $N_{s}$ is the number of cells in series, $N_{p}$ is the number of cells in parallel, Isc is the charge/discharge current. Since only one single cell were modeled the number of cells in parallel and series was set to one. The voltage $\left(V_{1}\right)$ across the capacitor $C_{1}$ on the main cell can be described as:

$$
v_{1}=\frac{-C_{0}+\sqrt{C_{0}^{2}+2 C v} Q_{1}}{C v}
$$

The capacitor $\mathrm{C} 1$ depends on the voltage $v_{1}$ and can be expressed as:

$$
C_{1}=C_{0}+C v v_{1}
$$

Where $\mathrm{C}_{0}$ is the constant capacitance in Farads $(\mathrm{F})$ and $C v$ is the constant parameter $(\mathrm{F} / \mathrm{V})$. Where $Q_{1}$ is the instantaneous charge of $C_{1}$ and can be calculated by:

$$
Q_{1}=C_{0} V_{1}+\frac{1}{2} C_{V} V_{1}^{2}
$$

The slow branch determines the internal energy distribution at the end of the charge or discharge cycle in the time range of minutes. The parallel resistance $\mathrm{R}$ describes the leakage current can be neglected for fast charge and discharge cycles. Concerning the slow cell, the voltage $v_{2}$ in the secondary capacity $C_{2}$ can be expressed by:

$$
v_{2}=1 / c_{2} \int i_{2} d t=1 / c_{2} \int \frac{1}{R_{2}}\left(v_{1}-v_{2}\right) d t
$$

Let $Q_{2}$ the instantaneous charge of $C_{2}$, we have:

$$
Q_{2}=\int i_{2} d t
$$

The current $i_{2}$ going in the secondary capacitor $C_{2}$ represented by:

$$
i_{2}=I s_{C}-i_{1}
$$

The current $i_{1}$ going in the main capacitor $C_{2}$ is expressed as:

$$
i_{1}=C_{1} \frac{d v_{1}}{d t}=\frac{d Q_{1}}{d t}=\left(C_{0}+C_{v} V_{1}\right) \frac{d v_{1}}{d t}
$$

\subsection{Paramteric experimental strcuture}

The internal parameters of the supercapacitor are the resistance and capacitance during transient response and they are undefined. In order toidentify those parameters, the following experiment has been set as in Figure 3. 


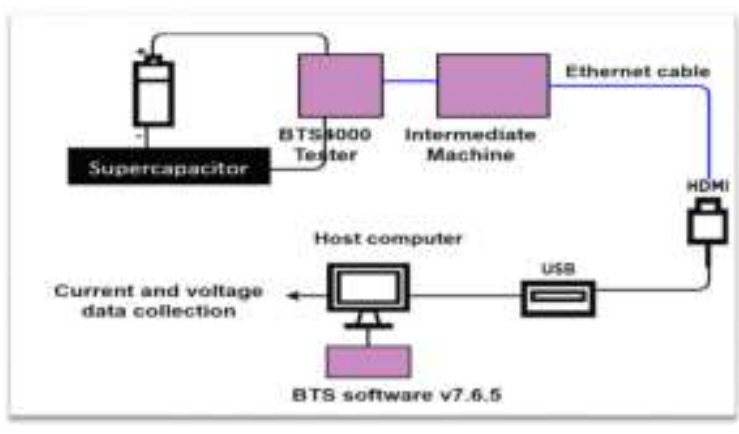

(a)

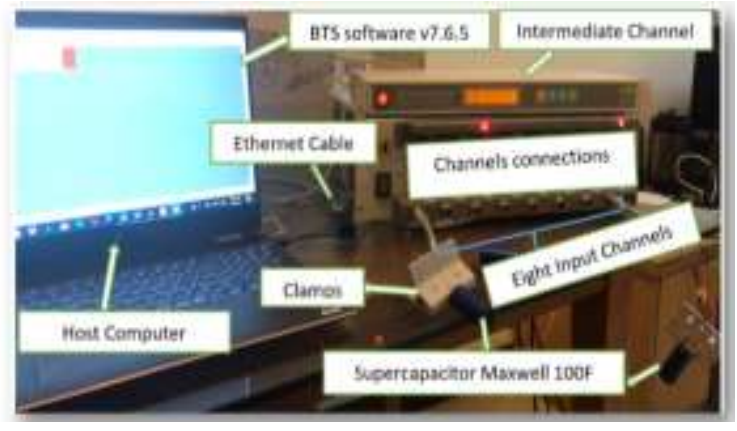

(b)

Figure 2. These figures are; (a) schematic diagram of the experimental set up, (b) experimental set up

The experimental set up was based on neware testing device with eight independent channels and intermediate machine, host computer with the neware software v7.5.6 and Matlab 2019a. The tester has the function of charge and discharge by applying certain software coding algorithm to the Neware software installed in the host computer as in Figure 3. The neware internal tester has two switches to operate in either charge or discharge based on the data collected from the current and voltage sensors circuits through DAQ, so transmits the control commands through the software to the SC connected to it .

The tester works on the range of $(0-10 \mathrm{~V})$ and current of $(0-6 \mathrm{~A})$, with sampling frequency of $1 \mathrm{~Hz}$ and measuring errors of less than $0.5 \%$. The middle machine has the functions of the following;network connections, receiving the control commands from the host computer, control the battery cycler and uploading the acquired data from the real time experiment. The computer has the function of controlling the cycler through the ethernet cables and store the data in the software from voltage and current sensors. The SC used in this experiment is MaxwellBCP100F and its important parameters are summarized in Table 1.

Table 1. supercapacitor parameters

\begin{tabular}{ccccc}
\hline Rated Voltage V & Rated Capacitance F & Typical ESRDC, M $\omega$ & Maximum Leakage Current mA & Maximum Peak Current A \\
\hline 2.7 & 100 & 8 & 0.26 & 61 \\
\hline
\end{tabular}

\subsection{Proposed method of parameters identification}

The internal paramters of the supercapacitor represent the resistance and capacitance in charge and discharge phases as two branches elements. The basic nonlinear relationship between charge, current and voltage can be expressed [6] as follow :

$$
i=\frac{d q}{d t} * \frac{d v}{d t}=\left(C_{O}+K_{V} v\right) \frac{d v}{d t}
$$

Integerting equation with respect to time:

$$
\int_{0}^{t} i d t=\int_{0}^{t}\left(C_{O}+K_{V} v\right) \frac{d v}{d t} d t
$$

Since the current is constant yields:

$$
t=f(v)=\frac{C_{O}}{I_{C}} v+\frac{1}{2} K_{V} \frac{1}{I_{C}} v^{2}
$$

Since the two-branch model have two capacitances in (11) we can assume that the values of those constants represented from (11) by:

$$
\begin{aligned}
& \mathrm{C}_{1}=\frac{C_{O}}{I_{C}} \\
& \mathrm{C}_{2}=\frac{K_{v}}{2 * I_{C}}
\end{aligned}
$$

So rearranging in (11)-(13) yields the following: 


$$
t=\mathrm{C}_{1} v+\mathrm{C}_{2} v^{2}
$$

Based on (14) the voltage during charging phase has two unknown coefficient $\mathrm{C} 1$ and $\mathrm{C} 2$. Based on voltage data obtained experimentally the values of these two coefficients $\mathrm{C} 1$ and $\mathrm{C} 2$ can be compared with (14) as displayed in Figure 4. From figure there is minues sign on the displayed equation in Figure 4 and the capacitance cannot negative rather than confirm the relationship physically, so that the two points have been selected for obtaining the values of $\mathrm{C} 1$ and $\mathrm{C} 2$ as in Figure 5.

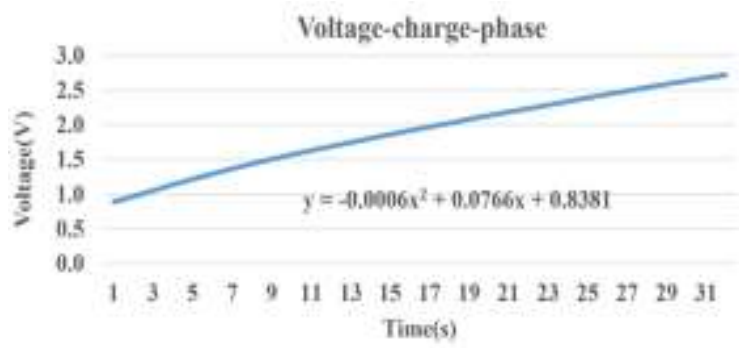

Figure 3. Experimental charging phase data

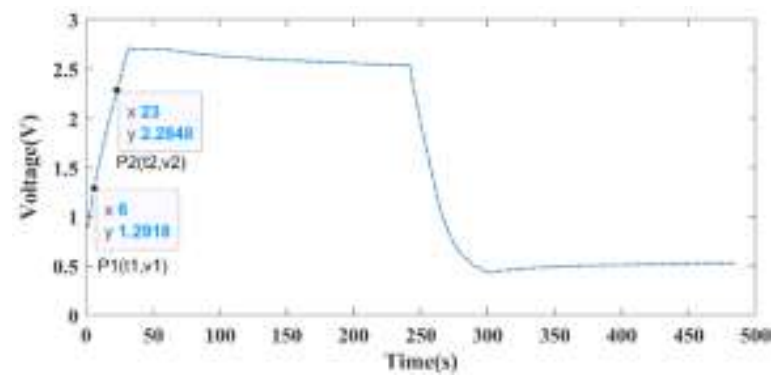

Figure 4. Experimental terminal voltage data of supercapacitor

Based on Figure 5, two points of charging phase were selected to calculate the values of immediate branch during charging phase. The selected points were based on the characteristics of SC specified on data sheet that the presentation of capacitances can be picked at $0.5,0.95$ of SC rated voltage or $0.4,0.8$ of rated voltage. Based on (14), the two points in Figure 5 must be equal since they are in the same charging phase as follow:

$$
\begin{aligned}
& \mathrm{t}_{1}=\mathrm{C}_{1} v 1+\mathrm{C}_{2} v 1^{2} \\
& \mathrm{t}_{2}=\mathrm{C}_{1} v 2+\mathrm{C}_{2} v 2^{2}
\end{aligned}
$$

By solving in (15) and (16) yields:

$$
\begin{aligned}
& C_{1}=\left[\frac{v_{1} * t_{2}-v_{2} * t_{1}}{v_{1} * v_{2}^{2}-v_{2} * v_{1}^{2}}\right] * I_{\text {charge }} \\
& C_{2}=\left[\frac{v_{1} * t_{2}-v_{1} * t_{1}}{v_{1} * v_{2}^{2}-v_{2} * v_{1}^{2}}\right] * I_{\text {charge }}
\end{aligned}
$$

So based on the two points obtained, the values of $C_{1}$ is $32.7604 \mathrm{~F}$ and $C_{2} 44.9576 \mathrm{~F}$. The resistance $\mathrm{R} 1$ is calculated based on:

$$
\mathrm{R}_{1}=\frac{\mathrm{D}_{\mathrm{v}}}{\mathrm{I}_{\text {charge }}}
$$

$\mathrm{D}_{\mathrm{v}}$ is the discharge drop voltage in the first $10 \mathrm{~ms}$ of the voltage discharge represented as follows:

$$
D_{v}=\frac{v_{1}-v_{2}}{t_{1}-t_{2}}
$$

$\mathrm{R}_{1}$ obtained as $0.0097 \mathrm{Ohm}$, so that the only parameter left is $\mathrm{R}_{2}$ by applying fitting curve applicatyion in matlab for terminal voltage during rest the exponential function can be expreesed in (21). The open cicuit voltage $(\mathrm{OCV})$ during discharge in the second phase displayed in Figure 6.

$$
O C V=a_{*} e^{-\frac{t}{\tau}}
$$

From Figure 6 the Matlab fitting curve equation with RMSE error of $0.008204 \mathrm{~V}$ reprented as:

$$
O C V=0.5017_{*} e^{-0.04139 t}
$$


So that the model was obtained and and time constant $\left(\tau_{2}\right)$ obtained by comparing (22) and (21), so $\mathrm{R}_{2}$ can be calculated based on the following (23). The all obtained internal parameters calculated and tabulated in Table 2.

$$
\begin{aligned}
& \tau_{2}=C_{2 *} R_{2} \\
& R_{2}=\frac{\tau_{2}}{c_{2}}
\end{aligned}
$$

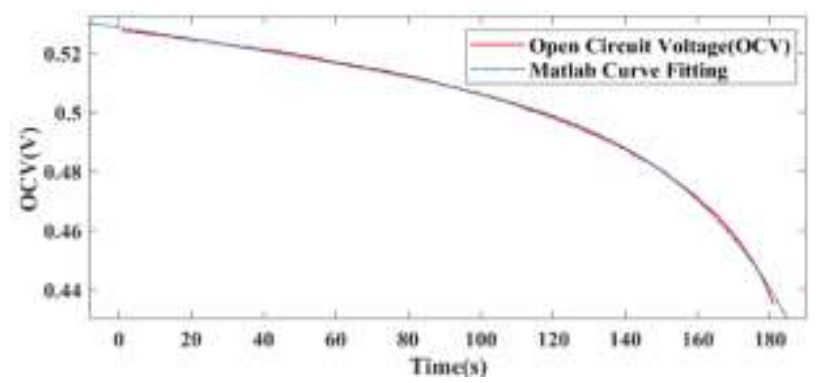

Table 2. Identification results of the two-branch equivalent circuit model

\begin{tabular}{ccccc}
\hline$R_{1} \mathrm{Ohm}$ & $C_{1} \mathrm{~F}$ & $C_{2} F$ & $R_{2} \mathrm{Ohm}$ & $\mathrm{Kv}$ \\
\hline 0.0097 & 32.760 & 44.9576 & 0.0008 & 10.9201 \\
4 & & & \\
\hline
\end{tabular}

Figure 5. Terminal volatge during discharge curve

\subsection{Simulation model}

After obtaining the identified parameters using the test procedures, the simulation model was built via Matlab Simulink. Line transmission model was used to be compared with the model proposed in this paper and finally the result was verified with experimental terminal voltage data of SC. The two-branch models, line transmission model and measured voltage are shown in Figure 7. Different experiments with different current profile were used in simulation to validate the the two-branch model. The detailed model of the two-branch model used in simulation is displayed precisely in Figure 8.

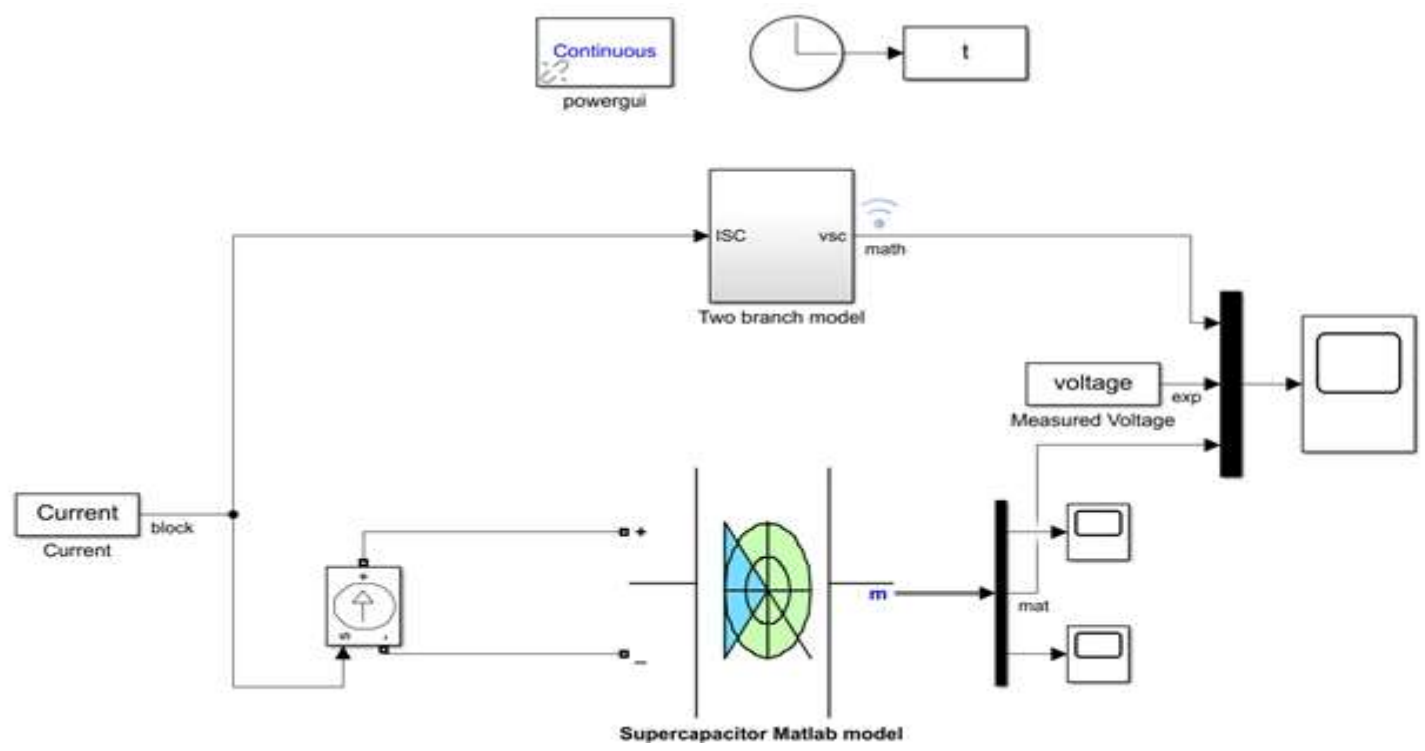

Figure 6. Matlab model and two branch model simulation

The two-branch model was based on the basic principle of Kirchhoff laws using (1)-(8). The number of parallel and series cells was set to one for the purpose of cell investigating model. The mathematical representation in (1)-(8) were used to calculate the terminal voltage as main parameters of supercapacitor model.current profile data were implemented in simulation based on extracted experimental data from neware. Also terminal voltage data from experiments exported to workspace in Matlab so that it will be used 
in Matlab Simulink for model validation and comparison of supercapacitor. The simulation model in Figure 7 and 8 used different profiles for different validation purpose which will be highlighted and discussed in results and discussion.

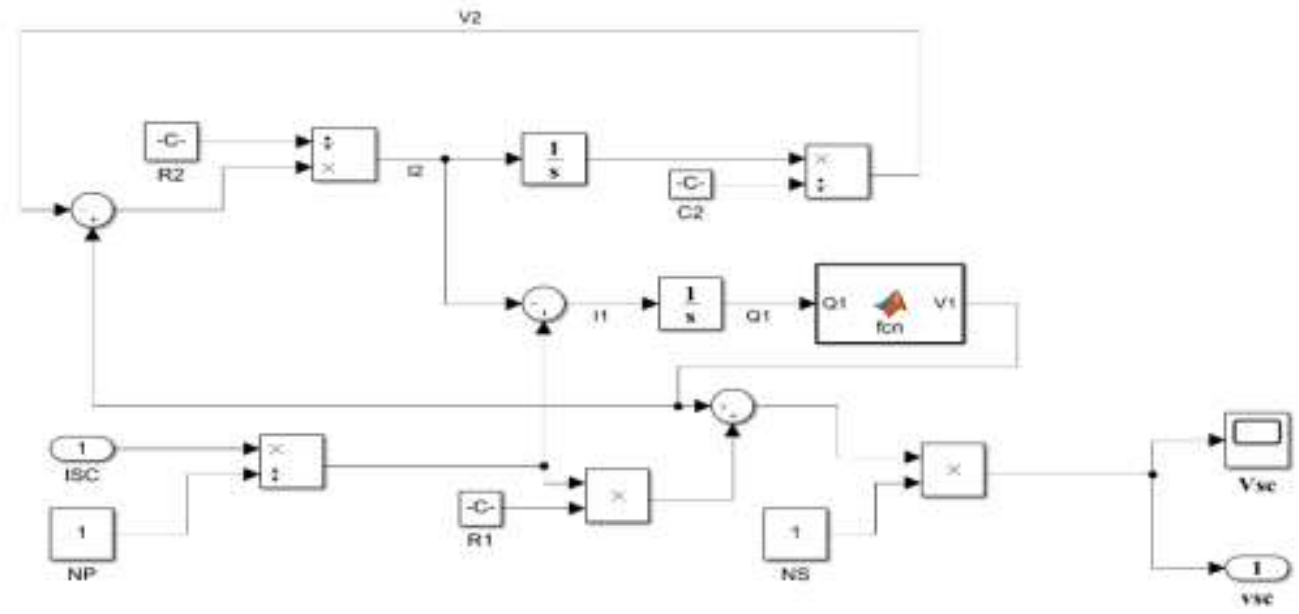

Figure 7. Subsystem Simulink for supercapacitor two branch model

\section{RESULTS}

The model validation mainly focused on the terminal voltage of supercpacitor as regulator parameter for controller design purposes. Different current profile used to testify the model validaity with the comaprison of Matlab built in model and measured voltage data. The first test used to charge/discharge the supercapacitors for two repeated cycles of rest-charge-discharge as shown in Figure 9.

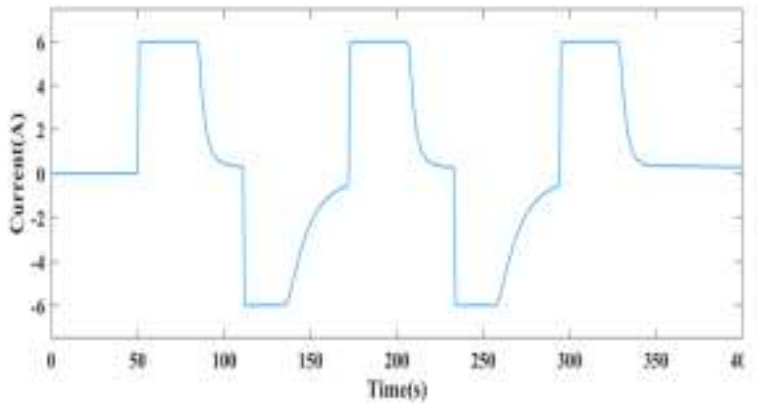

(a)

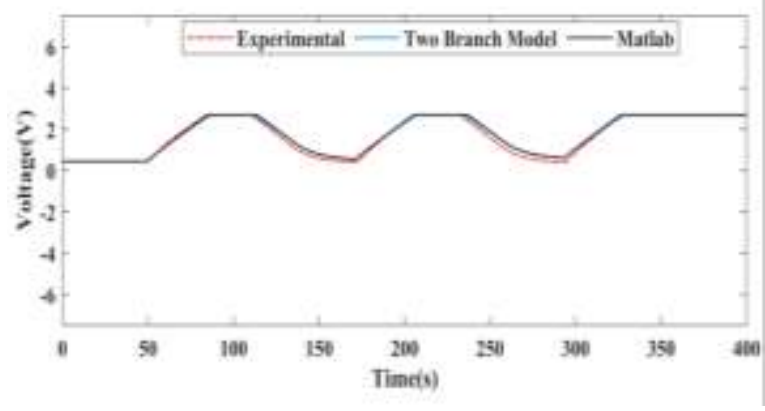

(b)

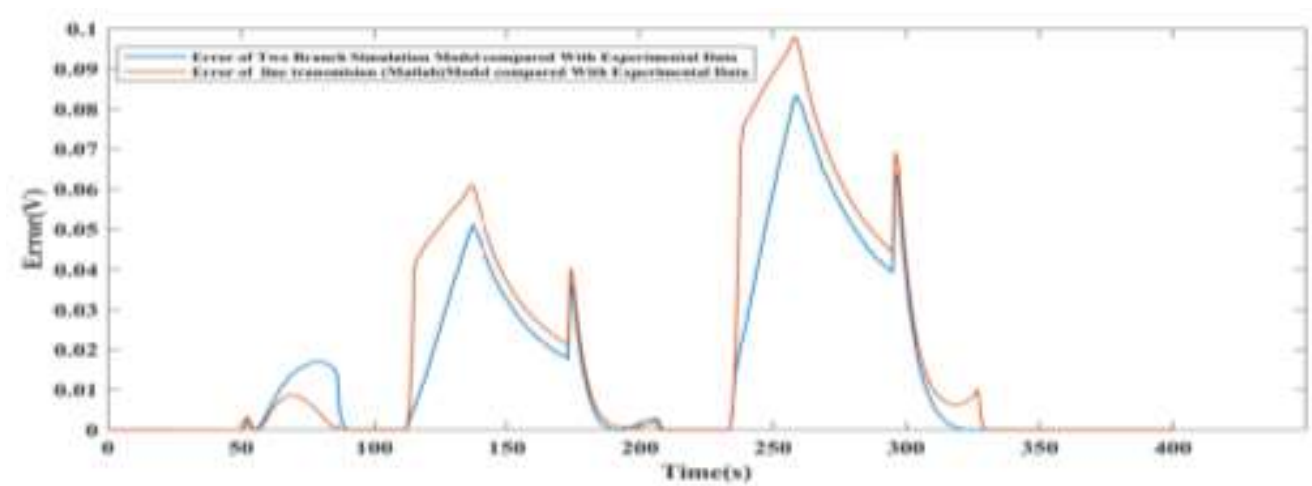

(c)

Figure 8. These figures are; (a) current profile (b) supercapacitor experimental terminal voltage data, simulation two branch model and simulation of line transmision model (c) error between measured and simulated models

Experimental design for an enhanced parametric modeling of supercapacitor... (Ali Mohsen Alsabari) 
The current profile in Figure 9(a) was set in neware device with complete cycles of (restcharge/discharge-rest) for the purpose of supercapacitor terminal voltage validation. The simulation started with period rest of 50 seconds to check self-discharge of supercapacitor with initial voltage measurement. Then, followed by 30 seconds of constant charging phase until it reached the rated voltage of $2.7 \mathrm{~V}$. After that, the SC was in rest for 30 seconds followed by one minute constant discharge phase .Furthermore the same time frame and phases for the first cycle was repeated. In Figure 9(b) the terminal voltage of supercapacitor (during rest-charging/discharging) phases for two cycles was compared with experimental curve data. The initial voltage was about $0.4427 \mathrm{~V}$ since supercapacitor cannot reached to zero voltage which confirms the selfdischarge property of supercapacitor. In Figure 9(b) by looking to the dotted red, blue and black lines which represent experimental data, two branch simulation model model respectively, it is clear that all models have similarity in trend but different prececision. The relative error was shown in Figure 9(c). for measured and simulated terminal voltage of two branch and and line transimision (Matlab) model. Of supercapacitor. The two branch model studied in this paper have a maximum relative error of $0.08 \mathrm{~V}$ while the line transimision (Matlab) model reached up to $0.1 \mathrm{~V}$. Based on relative error values, the two branch model have better terminal voltage response and followed measured data. Another parmater of charge/discharge validation of supercapacitor is load fluctuation for fast charge and discharge as displayed in Figure 10.

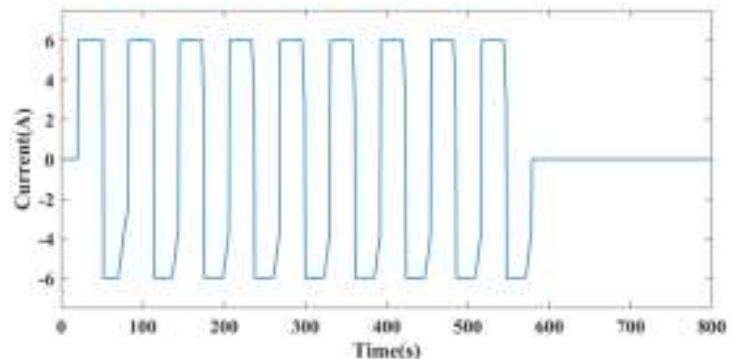

(a)

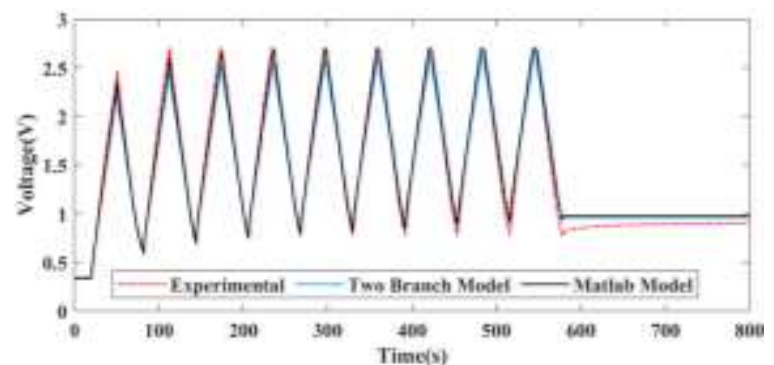

(b)

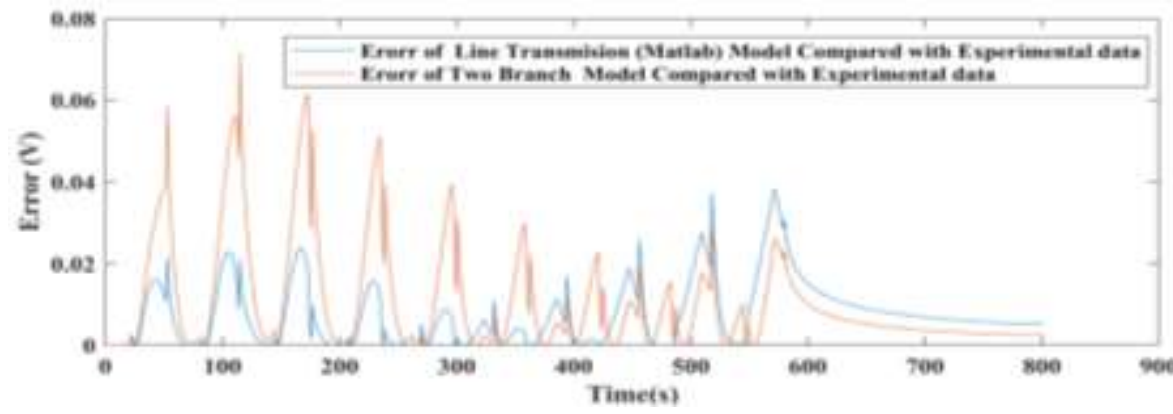

(c)

Figure 9. These figures are; (a) current profile, (b) supercapacitor experimental terminal voltage data, simulation two branch model and simulation of line transmision model, (c) error between measured and simulated models

As shown in Figure 10(a), the current profile used was by initiating the supercapacitor to rest for 20 seconds and then followed by charge and discharge for 30 seconds in each phase of charging and discharging. In Figure 10(b) it is clearly shown that during charging and discharging phase the terminal voltage compromised the measured voltage data of terminal voltage of supercapacitor. As in Figure 10(c), the maximum relative error for Matlab model was up to almost $0.075 \mathrm{~V}$ while the two-branch model investigated in this study was up to almost $0.04 \mathrm{~V}$. From the results obtained, the simulated two branch model shows better accuracy for terminal voltage response of supercapacitor. Another method for selfdischarge checking of supercapacitor within short period of time is displayed in Figure 10.

The rest/discharge as shown in Figure 11(a) the current profile of 6A of continuous current was used to discharge the supercapacitor. The supercapacitor was under rest while the supercapacitor was in fully charged phase then discharged for 30 seconds and being in rest for the remain simulation time of 30 seconds. In Figure 11(b) the two branch simulation model and experimental data of terminal voltage of supercapacitor 
were compared and validated. The error between the simulated two branch model and measured data have maximum relative error value of almost $0.02 \mathrm{~V}$ as shown in Figure 11(c).

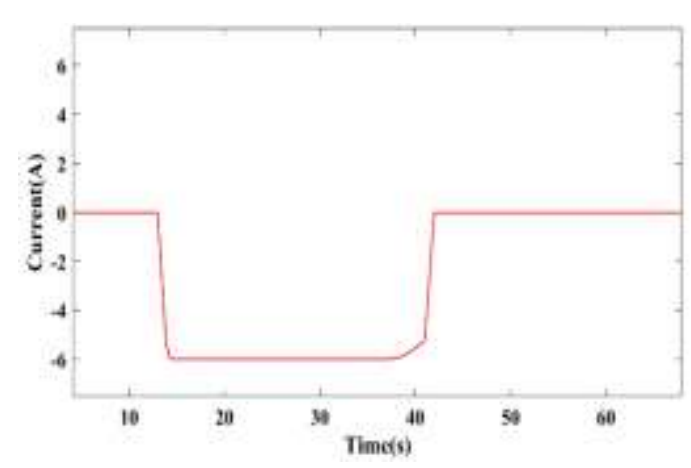

(a)

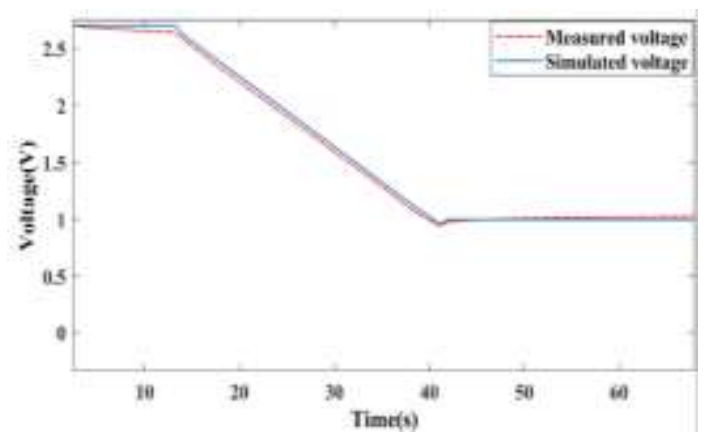

(b)

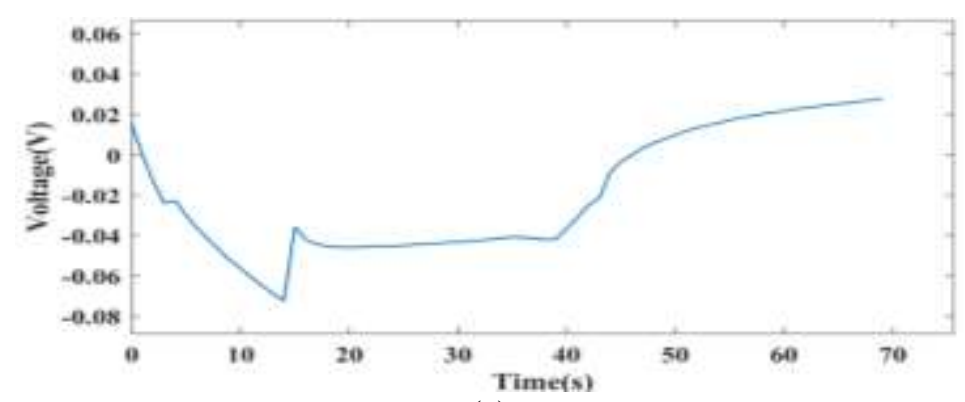

(c)

Figure 10. These figures are; (a) current profile (b) rest-discharging terminal voltage validation (c) error for rest and discharge validation of supercapacitor voltage

\section{DISCUSSION}

In over all, the investigated paramaters of the model of supercapacitor differs with regard to the application of the model itself but still accuracy of the model considered as main factor for model evaluation.in electrical model, the model accuracy depends on the identification profile, as well as the robustness of supercapacitor voltage response. In this work, the identification process was based on proposed empirical equations that showed the effectiveness of determining the internal paramters of supercapacitor successfully. The procedure proposed to evaluate the paramters have two measurements points in charge curve instead of four measurements used in charge and discharge phases in literature. These two points cover as much of the charging phase to represent mathematical equations of terminal voltage with capturing the physics relationship for resistance and capacitance. These results of internal paramters obtained are more precise by looking into the error evaluated in results section.

To add more, in this paper only one device neware BTS4000 used for modelling procedures which has the ability to capture many important data whilst is used to charge and discharge the SC compared to typical methods.Typical method need to use oscillcope, voltmeter, shunt board, data collection device, interface devices for host computer which may lead to the increase of the error in terminal voltage of SC.

By Looking to the literature many studied and investigated the model of supercapacitor widely, however the accuracy of the model have different factors for evaluation. This study compared and evaluated the model studied here based on tools used for modelling and accuracy error analysis. Taking some previous modelling work such as the study done by [26] in which their maximum relative error was up to almost $2 \%$.

Also another study done by [6] too many hardware tools used and their maximum relative error of terminal voltage was $0.25 \mathrm{~V}$. Besdies, the study done by [10] introduced the modeling of supercapacitor with improved two-branch circuit model by adding current controlled source to improve self-discharge rate of supercapacitor voltage. In this study they used neware testing device but the maximum relative errors ranged from 0.19 and up to $2.35 \%$ for similar experiments test profiles applied in this work. However, in this paper the maximum relative error was only up to $0.045 \mathrm{~V}$ for terminal voltage validation of supercapacitor.

Moreover [10] in their study the self-discharge rate was improved in their model but still there are long and complex method of the identification process of internal parameters by using filters and 
optimization algorthims. However in this study, the self-discharge rate during rest was relatively low and better than line transmission (Matlab) model with small variation error, so that it is not modelled as main parameters in this paper. In addition [28] stated in their study that it is difficult to have a unified standard to compare the self-discharge rates due to the different systems (including electrode materials, electrolytes, separators, and initial cell voltages) employed by researchers for self-discharge tests. However, due to the phenomenon of supercaictor self-discharge in practical applications once the supercacitor application requires being in rest for days and long time without charge or discharge, it is recommended for modeling self discharge accurately. To conclude, based on the error analaysis, the model investigated here has better error value which confirms that the tools and method used for internal paramters estimation and validation are recommended method for supercapacitor modelling.

\section{CONCLUSION}

The two-branch circuit model was investigated in different cureent profiles and the model represented the dynamic behavior of supercapacitor. Internal parameters of the model were identified and the simulation model was built via Matlab/Simulink successfully. The results of the simulation and measured voltage was compared and the results was comprmised. The validation error between measured and simulated models was investigate in each scenario discussed. The error was up to $0.04 \mathrm{~V}$ as maximum relative error which shows the effectiveness and validity of the model compared to the literature. Finally, the selfdischarge rate of supercapacitor was improved and followed the measured voltage. It is recommended for future work to study the effect of self-discharge rate during long period of rest in practical applications for using the two-branch circuit model. The neware testing device used mainly for battery testing and it can be used for supercapacitor as well for charge and discharge, however it is recommended to buy and use it if many cells not only one cell needed to be modelled for cost saving.

\section{ACKNOWLEDGEMENTS}

The authors would like to thank the Faculty of Engineering, University Putra Malaysia (UPM) for providing the Grant Vot 959100 and facilities and conducive learning environment in conducting the research.

\section{REFERENCES}

[1] Y. Parvini, J. Siegel, A. Stefanopoulou, and A. Vahidi, "Supercapacitor Electrical and Thermal Modeling, Identification, and Validation for a Wide Range of Temperature and Power Applications," IEEE Trans. Ind. Electron., vol. 63, no. 3, pp. 1574-1585, 2015, doi: 10.1109/TIE.2015.2494868.

[2] A. Berrueta, I. San Martín, A. Hernández, A. Ursúa, and P. Sanchis, "Electro-thermal modelling of a supercapacitor and experimental validation," J. Power Sources, vol. 259, pp. 154-165, 2014, doi: 10.1016/j.jpowsour.2014.02.089.

[3] K. Popoola, "Modelling and Simulation of Supercapacitor for Energy Storage Applications," vol. 7, no. 1, pp. 2-7, 2018.

[4] K. Lia, Y. Wahiba, and E. Evgueniy, "Hybrid battery/supercapacitor energy storage system for the electric vehicles," J. Power Sources, vol. 374, pp. 237-248, 2018, doi: 10.1016/j.jpowsour.2017.11.040.

[5] I. Jiya, N. Gurusinghe, and R. Gouws, "Electrical Circuit Modelling of Double Layer Capacitors for Power Electronics and Energy Storage Applications: A Review," Electronics, vol. 7, no. 11, p. 268, 2018, doi: 10.3390/electronics7110268.

[6] P. Saha and M. Khanra, "Equivalent circuit model of supercapacitor for self-discharge analysis - A comparative study," In. 2016 International Conference on Signal Processing, Communication, Power and Embedded System (SCOPES), 2016, pp. 1381-1386, doi: 10.1109/SCOPES.2016.7955667.

[7] A. Lahyani, P. Venet, A. Guermazi, and A. Troudi, "Battery/Supercapacitors Combination in Uninterruptible Power Supply (UPS)," IEEE Trans. Power Electron., vol. 28, no. 4, pp. 1509-1522, 2013, doi: 10.1109/TPEL.2012.2210736.

[8] L. Zhang, X. Hu, Z. Wang, F. Sun, and D. G. Dorrell, "A review of supercapacitor modeling, estimation, and applications: A control/management perspective," Renew. Sustain. Energy Rev., vol. 81, pp. 1868-1878, 2018, doi: 10.1016/j.rser.2017.05.283.

[9] L. E. Helseth, "Modelling supercapacitors using a dynamic equivalent circuit with a distribution of relaxation times," J. Energy Storage, vol. 25, p. 100912, 2019, doi: 10.1016/j.est.2019.100912.

[10] D. Xu, L. Zhang, B. Wang, and G. Ma, "Modeling of Supercapacitor Behavior With an Improved Two-Branch Equivalent Circuit,” IEEE Access, vol. 7, pp. 26379-26390, 2019, doi: 10.1109/ACCESS.2019.2901377.

[11] K. Liu, C. Zhu, R. Lu, and C. C. Chan, "Improved study of temperature dependence equivalent circuit model for supercapacitors," IEEE Trans. Plasma Sci., vol. 41, no. 5, pp. 1267-1271, 2013, doi: 10.1109/TPS.2013.2251363.

[12] Y. Wang, L. Wang, M. Li, and Z. Chen, "A review of key issues for control and management in battery and ultracapacitor hybrid energy storage systems," eTransportation, vol. 4, 2020, doi: 10.1016/j.etran.2020.100064. 
[13] M. C. Argyrou, P. Christodoulides, C. C. Marouchos, and S. A. Kalogirou, "Hybrid battery-supercapacitor mathematical modeling for PV application using Matlab/Simulink," In 2018 53rd International Universities Power Engineering Conference (UPEC), 2018, pp. 1-6, doi: 10.1109/UPEC.2018.8541933.

[14] S. M. Faresse, M. Assini, and A. Saad, "Hybrid energy storage system optimal sizing for urban electrical bus regarding battery thermal behavior," International Journal of Electrical and Computer Engineering (IJECE), vol. 10, no. 3, pp. 2894-2911, 2020, doi: 10.11591/ijece.v10i3.pp2894-2911.

[15] Z. Cabrane, M. Ouassaid, and M. Maaroufi, "Analysis and evaluation of battery-supercapacitor hybrid energy storage system for photovoltaic installation,” Int. J. Hydrogen Energy, vol. 41, no. 45, pp. 20897-20907, 2016, doi: 10.1016/j.ijhydene.2016.06.141.

[16] G. Navarro, J. Nájera, J. Torres, M. Blanco, M. Santos, and M. Lafoz, "Development and experimental validation of a supercapacitor frequency domain model for industrial energy applications considering dynamic behaviour at high frequencies," Energies, vol. 13, no. 5, 2020, doi: 10.3390/en13051156.

[17] A. Wangsupphaphol, N. R. N. Idris, A. Jusoh, N. D. Muhamad, and I. M. Alsofyani, "Energy and power control strategy for battery electric vehicle with supercapacitors," In 2014 IEEE Conference on Energy Conversion (CENCON), 2014, pp. 13-18, doi: 10.1109/CENCON.2014.6967469.

[18] C. Zheng, W. Li, and Q. Liang, "An Energy Management Strategy of Hybrid Energy Storage Systems for Electric Vehicle Applications," IEEE Transactions on Sustainable Energy, vol. 9, no. 4, pp. 1880-1888, 2018, doi: 10.1109/TSTE.2018.2818259.

[19] A. Mansour, C. Mohamed Hedi, and B. Faouzi, "Experimental Study of a Pack of Supercapacitors Used in Electric Vehicles,” The Scientific World Journal, vol. 2017, 2017, doi: 10.1155/2017/6702838.

[20] C. Xie, X. Liu, L. Huang, and W. Fang, "Modeling of supercapacitor modules in the hybrid powertrain with a nonlinear 3-branch equivalent circuit," International Journal of Energy Research, vol. 42, no. 11, pp. 3524-3534, 2018, doi: 10.1002/er.4094.

[21] W. Jing, C. H. Lai, W. S. H. Wong, and M. L. D. Wong, "A comprehensive study of battery-supercapacitor hybrid energy storage system for standalone PV power system in rural electrification," Applied Energy, vol. 224, pp. 340356, 2018, doi: 10.1016/j.apenergy.2018.04.106.

[22] V. Musolino, L. Piegari, and E. Tironi, "New full-frequency-range supercapacitor model with easy identification procedure," IEEE Trans. Ind. Electron., vol. 60, no. 1, pp. 112-120, 2013, doi: 10.1109/TIE.2012.2187412.

[23] Pezzolato, S., "Modeling And Model Validation Of Supercapacitors For Real-Time Simulations," Doctoral dissertation, Alma Mater Studiorum-Università Di Bologna, Italy, 2019.

[24] L. Zhang, "Modelling and State-of-Charge Estimation for Ultracapacitors in Electric Vehicles," Doctoral dissertation, Fact. of Eng. and Inf. Tech., Univ. of Technology, Sydney, Australia, 2016.

[25] L. Zhang, Z. Wang, X. Hu, F. Sun, and D. G. Dorrell, "A comparative study of equivalent circuit models of ultracapacitors for electric vehicles," Journal of Power Sources, vol. 274, pp. 899-906, 2015, doi: 10.1016/j.jpowsour.2014.10.170.

[26] T. Mesbahi, N. Rizoug, F. Khenfri, P. Bartholomeüs, and P. Le Moigne, "Dynamical modelling and emulation of Li-ion batteries-supercapacitors hybrid power supply for electric vehicle applications," IET Electr. Syst. Transp., vol. 7, no. 2, pp. 161-169, 2017, doi: 10.1049/iet-est.2016.0040.

[27] W. Zhang et al., "Self-discharge of supercapacitors based on carbon nanotubes with different diameters," Electrochim. Acta, vol. 357, p. 136855, 2020, doi: 10.1016/j.electacta.2020.136855.

\section{BIOGRAPHIES OF AUTHORS}

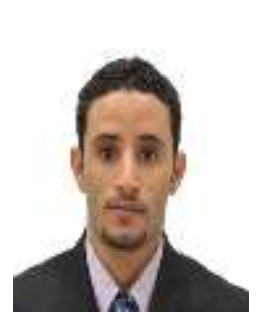

Ali alsabari was born in Yemen 1st January 1986. He received his bachelor degree in mechatronics Engineering from International Islamic University Malaysia. (IIUM), Malaysia in 2013. He later received his master degree from University Technology Malaysia (UTM), SkudaiJohor, Malaysia in 2016. He is now under scholarship from Malaysian government studying PhD in his sixth semester in control system engineering in University Putra Malaysia (UPM) Malaysia

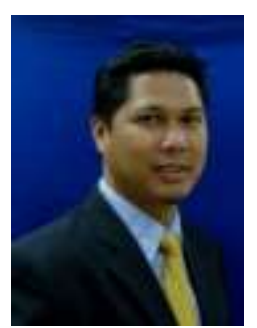

Mohd. Khair Hassan was born in Malacca, Malaysia. He received the B.Eng. degree in Electrical and Electronic Engineering from the University of Portsmouth, U.K., in 1998, the M.Eng. degree in Electrical Engineering from Universiti Teknologi Malaysia (UTM), Skudai, Johor, Malaysia, in 2001, and the Ph.D. degree in Automotive Engineering from Universiti Putra Malaysia (UPM), Serdang, Selangor, Malaysia, in 2011. He is currently an Associate Professor and Head of the Department of Electrical and Electronic Engineering, UPM. His area of interests includes control system, automotive control, electric vehicle, and AI applications. Currently, his focuses are on $\mathrm{x}$-by-wire technology and optimal strategy for energy consumption in electric vehicle. Dr. M.K. Hassan is a Professional Engineer registered under Board of Engineers Malaysia (BEM) and Vice-Precident for Soceity of Malaysia Automatic Control Engineer, MACE-IFAC. 


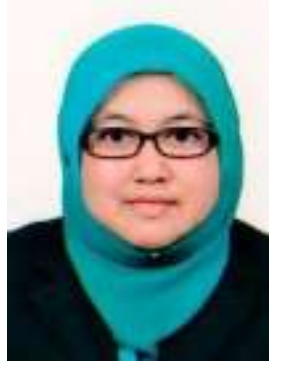

Dr. Azura Che Soh is an Associate Professor at the Department of Electrical and Electronic Engineering, Faculty of Engineering, University Putra Malaysia Serdang (UPM), Selangor. She graduated in BEng in Electronic/Computer in 1999 and MSc (Electrical \& Electronic of Engineering) in 2002 from Universiti Putra Malaysia. She obtained PhD (Electrical Engineering) from Universiti Teknologi Malaysia in 2011. She was main researcher of Control System and Signal Processing (CSSP) Research Center, Department of Electrical and Electronics Engineering, UPM. She was a coordinator for Control and Automation of Laboratory and Associate Researcher at MYAGEING institute. She is now supervising $3 \mathrm{PhD}, 6 \mathrm{MSc}$ directly under her supervision including $10 \mathrm{PhD}$ and $16 \mathrm{MSc}$ co-supervision postgraduate students. She published 64 journals and 69 proceedings. Her research interests are intelligent control system, control system and system modelling.

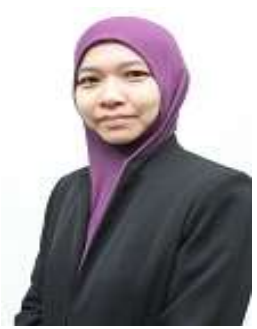

Ribhan Zafira Binti Abdul Rahman is Dr in Department. Of Electrical and Electronic Engineering, Faculty of Engineering, Universiti Putra Malaysia 43400 UPM Serdang, Selangor. Her phD in Electrical Engineering Universiti Teknologi Malaysia, Malaysia was in 2013. Electrical M.E. in Electrical Eng. Universiti Teknologi Malaysia, Malaysia in 2001. Electrical B.Eng. (Hons) in Electrical and Electronics Engineering Liverpool John Moores University, UK in 1998. Electrical and Electronics Diploma In Electrical And Electronic Engineering Institut Teknologi Mara, Malaysia in 1995. 